\title{
Inducible costimulator is essential for collagen-induced arthritis
}

Nurieva, R.I., Treuting, P., Duong, J., Flavell, R.A., and Dong, C. 2003.

J. Clin. Invest. 111:701-706. doi:10.1172/JCI200317321.

My colleagues and I performed collagen-induced arthritis experiments reported in the paper under the impression that this study had been approved by the Institutional Animal Care and Usage Committee (IACUC) of the University of Washington, but we were subsequently informed by this committee that such approval had not been granted. There were no scientific errors in the paper, and we stand by the validity, importance, and interest of the results. However, the paper is to be retracted according to the JCI's policy of publishing only studies that have been formally approved by an IACUC. We are sorry for any inconvenience this has caused.

Chen Dong 